\title{
Análisis bibliométrico de The European Journal of Psychology Applied to Legal Context entre 2009-2018
}

\section{Bibliometric analysis of The European Journal of Psychology Applied to Legal Context between 2009-2018}

\author{
Elisa Alfaro ${ }^{1}$, Francisco González-Sala ${ }^{2}$, Macarena Tortosa ${ }^{3}$, Julia Osca ${ }^{4}$ \\ ${ }^{1}$ Universidad de Valencia.m.elisa.alfaro@uv.es \\ ${ }^{2}$ Universidad de Valencia.francisco.gonzalez-sala@uv.es \\ ${ }^{3}$ Universidad Internacional de Valencia-VIU. macarena.torrosa@hotmail.com \\ ${ }^{4}$ INGENIO (CSIC-UPV), Universitat Politècnica de València. m.julia.osca@uv.es
}

Recibido: 30/9/2019

Aceptado: 9/12/2019

\section{Copyright $(C)$}

Facultad de CC. de la Educación y Deporte. Universidad de Vigo

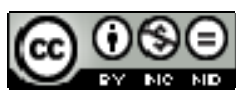

Dirección de contacto:

Francisco González-Sala

Facultad de Psicología, Universidad de

Valencia

Avenida Blasco Ibáñez, 21

46010 VALENCIA

\begin{abstract}
Resumen
El estudio realiza un análisis bibliométrico del European Journal of Psychology Applied to Legal Context entre 2009-2018, situado en el percentil 96 en su categoría. Se construyó una base de datos que permite analizar autores, instituciones, países, género, temáticas e impacto en las bases de datos de la Web of Science. Los resultados permiten observar el incremento de la colaboración internacional, y la existencia de un grupo reducido de autores muy productivos, en grupos de colaboración próximos al equipo editorial y a la Sociedad Española de Psicología Jurídica y Forense, de la que la revista es órgano de expresión. Encuentra un eje vertebrador institucional que incluye un pequeño grupo de universidades españolas, y que vincula universidades y centros extranjeros, sobre todo mejicanos y de la Europa del Norte. Hay una elevada presencia de mujeres, productivas, con buen posicionamiento en el orden de firma e impacto diferencial en la comunidad científica, aunque no en el Consejo Editorial. Son temas destacados la evaluación, la credibilidad y el testimonio. El 95\% de sus artículos han sido citados, y sólo siete explican una cuarta parte del total de citas recibidas. La revista está bien posicionada en el ámbito de las aplicaciones de la Psicología al contexto legal.
\end{abstract}

\section{Palabras Clave}

Revista de Psicología, Bibliometría, European Journal of Psychology Applied to Legal Context, Internacionalización

\begin{abstract}
The aim of this study was to carry out a bibliometric analysis of "The European Journal of Psychology Applied to Legal Context", which recently reached the 96th percentile in its category. A database was built to analyze authors, institutions, countries, gender, themes and impact on the databases of the Web of Science. The results allowed us to observe the increase in the international collaboration in the period 2009-2018. In this sense, it has been found that there is a small group of very productive researchers, linked to consolidated research groups, close to the editorial team and the Spanish Society of Legal and Forensic Psychology, from which the
\end{abstract}


journal constitutes an expression body. Furthermore, an institutional backbone, that includes a small group of Spanish universities, and also links foreign universities, especially Mexican and Northern European universities, was found. There is a high presence of female authors, many of them very productive, with a good positioning in the order of signature and differential impact among the scientific community, although not to the date in the Editorial Board. Likewise, it was found that the largest number of published works was focused on the areas of psychological evaluation, credibility and testimony. $95 \%$ of the articles have been currently cited, and only seven of them explain a quarter of the total number of citations received by the journal. The journal occupies a very prominent place in the field and is being consolidated as a key source for the dissemination of research in legal psychology.

\section{Key Words}

Journal of Psychology, Bibliometrics, European Journal of Psychology Applied to Legal Context, Internationalization

\section{INTRODUCCIÓN}

La investigación científica solo tiene razón de ser desde el momento en que el autor comunica sus resultados al resto de la comunidad científica a través de una publicación con cierta capacidad de difusión y al alcance de toda la comunidad investigadora interesada en el tema. Las publicaciones científicas recogen el producto final de la investigación, y sus artículos constituyen el principal elemento de valoración en las carreras profesionales de los investigadores (Aguado-López y Becerril-García, 2016, Osca-Lluch, Tortosa, González-Sala y Tortosa, 2017).

La Psicología Jurídica en España es una disciplina relativamente joven (Tortosa, Civera, Fariña y Alfaro, 2008), que experimentó un gran desarrollo en la década de 1980 (Fariña, Arce y Jölluskin, 2000; Fariña, Arce y Novo, 2005), periodo en el que se puso de manifiesto la multidisciplinariedad de la materia, considerándose como un área con estatus propio (Carpintero, 2006). No obstante, fue en la última década del pasado siglo cuando la Psicología Jurídica inició una rápida expansión en España, al igual que en el resto de Europa y América (Fariña, Arce y Seijo, 2005).

El Colegio de Psicólogos de España inicia un pionero Anuario de Psicología Jurídica (1990), que venía a unirse a la fugaz (1989-1991) revista Delincuencia. Es esta la década en la que se define y concreta el perfil profesional de la Psicología Jurídica (de Nicolás, 1995; Colegio Oficial de Psicólogos, 1998), con un inmediato éxito profesional (Santolaya, Berdullas, y Fernández, 2001). Pronto fue recogido en materias propias del perfil en los currículos de las Universidades (Alfaro, Real, Tortosa y Jölluskin, 2004; Quevedo-Blasco, Ariza y Raya, 2012), donde ha acabado definiendo una oferta de postgrado en franco crecimiento (González-Sala, Osca-LLuch, Tortosa-Gil y Peñaranda-Ortega, 2018).

Se ha caracterizado el siglo XXI como una etapa de nuevos retos en la Psicología Jurídica (Arce y Fariña, 2013). Entre ellos su propia delimitación conceptual (Muñoz et al., 2011), la adecuada definición de los diferentes campos que la integran tanto a nivel de investigación como de desempeño profesional, la definitiva incorporación en los currículos universitarios, la vertebración mediante congresos periódicos, la regulación profesional, la internacionalización mediante políticas adecuadas de comunicación y el 
posicionamiento de revistas en índices de citas (Osca-Lluch, Tortosa, González-Sala, y Tortosa, 2017).

Forma parte de la respuesta a esos retos la creación de la Sociedad Española de Psicología Jurídica y Forense (SEPJF), la reunión periódica de académicos y profesionales interesados en congresos específicos sobre el ámbito abiertos a otras lenguas, y se inicia una revista especializada, The European Journal of Psychology Applied to Legal Context, coeditada con el COP Madrid desde 2013 (Anónimo, 2013), en lengua inglesa y orientada a internacionalizar y posicionar la Psicología Jurídica y Forense española.

\section{METODOLOGÍA}

El objetivo del artículo es realizar un análisis bibliométrico de la revista The European Journal of Psychology Applied to Legal Context (The EJPALC, en adelante). En el período analizado, es la única revista española de Psicología aplicada al contexto legal de perfil académico-profesional indexada en el JCR de la Web of Science, además de estarlo en SJR de Scopus (ver Tabla 1), siendo la revista española de Psicología mejor posicionada en ambos índices (Osca-Luch et al., 2017).

\begin{tabular}{lccccccccc}
\hline Áreas temáticas & $\mathbf{2 0 1 0}$ & $\mathbf{2 0 1 1}$ & $\mathbf{2 0 1 2}$ & $\mathbf{2 0 1 3}$ & $\mathbf{2 0 1 4}$ & $\mathbf{2 0 1 5}$ & $\mathbf{2 0 1 6}$ & $\mathbf{2 0 1 7}$ & $\mathbf{2 0 1 8}$ \\
\hline Applied Psychology (SJR) & Q4 & Q3 & Q2 & Q3 & Q3 & Q3 & Q2 & Q2 & Q1 \\
\hline Law (JCR) & & & Q3 & Q2 & Q1 & Q2 & Q1 & Q1 & Q1 \\
\hline Law (SJR) & Q3 & Q2 & Q2 & Q2 & Q1 & Q2 & Q1 & Q1 & Q1 \\
\hline Psychology Multidisciplinary (JCR) & & & Q3 & Q3 & Q3 & Q3 & Q2 & Q1 & Q1 \\
\hline
\end{tabular}

Nota. En 2009 no se computó el impacto en ninguna de las bases porque era el primer año de publicación de la revista y, por tanto, no era posible.

Tabla 1. Evolución del impacto de The EJPALC en las bases de datos SJR y JCR

Para la realización del estudio se ha creado una base de datos relacional, en la que se han recogido todos los trabajos publicados en la revista durante el período 2009-2018. Se ha procedido a la normalización de los datos de los autores e instituciones. Para cada uno de los trabajos se han identificado los autores firmantes de cada artículo, sexo del primer y último autor firmante, lugar de trabajo y el área temática en que se inscribe.

Se utilizaron indicadores bibliométricos relacionados con la evolución temporal de la producción, la distribución geográfica e institucional, los países con los que se colabora, los autores, su productividad y redes de colaboración, introduciendo una perspectiva de género, y la visibilidad de los artículos. En todos los casos, a excepción de la categoría "área temática" se verificaron los supuestos de análisis directamente por la información proporcionada por los propios autores en los documentos recogidos en la revista, realizándose verificaciones externas en los casos de duda. Para la obtención de indicadores de producción, se han revisado y normalizado los nombres de los autores y de las instituciones e identificado el sexo de cada firmante.

El análisis temático se llevó a cabo mediante una codificación independiente por parte de dos investigadores de reconocido prestigio, que actuaron como evaluadores “ciegos" a los objetivos del estudio. Cuando la contribución podía ser asignada a más de una categoría, se priorizó aquella que se adecuaba más a los objetivos principales de la 
investigación explicitados en el cuerpo del artículo, que se enviaba a ambos evaluadores. Aquellos trabajos que, a pesar de estas precauciones, no generaban acuerdo, fueron analizados por un tercer investigador también "ciego" a los objetivos del estudio, tomando la decisión de asignación por mayoría de acuerdo entre evaluadores.

\section{RESULTADOS}

\subsection{Productividad}

El número total de trabajos publicados por The EJPALC, que han sido indexados en la base de datos Web of Science (WoS) es de 105. De ellos, 104 son artículos originales y un trabajo es una breve editorial que fue publicada en el año 2010, y que no incluimos en los cálculos.

Los trabajos cuentan con un total de 341 firmas pertenecientes a 247 autores. De estos, solamente hay 3 autores $(1,20 \%)$ que pueden considerarse grandes productores al firmar más de 4 trabajos, ya que cada uno ha firmado en 7 artículos, 57 autores $(23,10 \%)$ pueden considerarse medianos productores porque han firmado entre 2 y 4 artículos, y 187 autores (75,70\%) que pueden considerarse ocasionales o transitorios, al haber firmado en un solo trabajo a lo largo del período analizado.

En la Tabla 2 se muestra la relación de autores que han firmado en 3 ó más artículos. Se observa que un pequeño subgrupo de autores, 20 investigadores $(8,09 \%)$ concentra una importante representación de las autorías de las publicaciones analizadas explicando el $22,28 \%$ de las firmas.

\begin{tabular}{|c|c|c|}
\hline Autores & Trabajos & Instituciones \\
\hline Expósito, Francisca & 7 & Univ. de Granada, España \\
\hline Gracia Fuster, Enrique & 7 & Univ. de Valencia, España \\
\hline Lila, Marisol & 7 & Univ. de Valencia, España \\
\hline Rodríguez-Díaz, Francisco Javier & 4 & Univ. de Oviedo, España \\
\hline Herrera, María del Carmen & 4 & Univ. de Granada, España \\
\hline Herrero, Juan & 4 & Univ. de Oviedo, España \\
\hline Ruiz-Hernández, José Antonio & 4 & Univ. de Murcia, España \\
\hline Amado, Bárbara G. & 3 & Centro Universitario de la Defensa, España \\
\hline Andrés Pueyo, Antonio & 3 & Univ. de Barcelona, España \\
\hline Arce, Ramón & 3 & Univ. de Santiago Compostela, España \\
\hline Fariña, Francisca & 3 & Univ. de Vigo, España \\
\hline Gómez-Fraguela, José Antonio & 3 & Univ. de Santiago Compostela, España \\
\hline Granhag, Pär Anders & 3 & Univ. de Gothenburg, Suecia \\
\hline Herrera, Antonio & 3 & Univ. de Granada, España \\
\hline Jiménez, Fernando & 3 & Univ. de Salamanca, España \\
\hline Llor-Esteban, Bartolomé & 3 & Univ. de Murcia, España \\
\hline Palmer, Alfonso & 3 & Univ. de Illes Balears, España \\
\hline Pérez, Beatriz & 3 & Univ. de la Frontera, Chile \\
\hline Sánchez, Guadalupe & 3 & Univ. de Salamanca, España \\
\hline Vilariño, Manuel & 3 & Univ. de Santiago Compostela, España \\
\hline
\end{tabular}

Tabla 2. Autores con 3 o más trabajos publicados 
De los 20 autores que han colaborado en la realización de tres o más artículos, 6 son mujeres y 14 hombres, una distribución de sexo asonante con la distribución global encontrada en la revista. Los autores más productivos son Francisca Expósito, Enrique Gracia y Marisol Lila, con 7 trabajos cada uno de ellos. Tres de ellos son o han sido miembros de la Junta Directiva de la SEPJF: Francisco Javier Rodríguez-Díaz (tesorero hasta 2018), Marisol Lila (Vocal de Psicología Penitenciaria) y Francisca Expósito (Vocal de Violencia de Género). Al analizar la distribución por sexo, de los 42 autores que han publicado 2 trabajos hay 22 mujeres y 20 hombres.

Los autores que publican en la revista están afiliados a 73 instituciones diferentes, el $31,50 \%$ españolas (23), aportando un mayor número de firmantes. Entre los 20 países de los que proceden los autores que publican en la revista, 12 son europeos, uno asiático, el Estado de Palestina, y los otros 7 pertenecen a América (Norte, Centro y Sur de América).

La revista recoge firmas de hasta 50 instituciones no españolas, sobre todo universidades, que explican el $92 \%$ de las mismas. Una destacable distribución diferencial, un $4 \%$ de las instituciones explica el $23,86 \%$ de las firmas, The University of Gothenburg, con 17 firmas, y The Southern Connecticut State University con 9, son las más representadas. Si añadimos la Universidad Pública de Leicester (England) y la Université d'Angers, sobre todo el Laboratoire de Psychologie des Pays de la Loire, se explica el $34 \%$ de las firmas extranjeras en la revista. Son 32, un $65,31 \%$, las instituciones de las que tan solo proviene una firma, y explican un 29,36\%. El resto porcentual lo explican 14 instituciones procedentes de Italia, EE.UU., Portugal, Suiza, México, Reino Unido, Alemania, Países Bajos, Noruega, Suecia y Rumania. Por continentes, es Europa el más representado (Suecia, Italia, Portugal, Francia, Suiza, Reino Unido, Alemania, Países Bajos, Noruega, Estonia y Rumania), luego el americano (EE.UU., México, Ecuador, Chile, Argentina, Perú y Canadá). El continente asiático está representado por la pública Al-Azhar University - Gaza.

La revista recoge firmas de hasta 23 instituciones españolas, sobre todo universidades, que explican el $87 \%$ de aquellas y contribuyen significativamente al posicionamiento en el ranking de investigación de las universidades públicas españolas (Buela-Casal, Guillén-Riquelme, Ramiro-Sánchez, y Quevedo-Blasco, 2017). Son las universidades de Santiago de Compostela, Barcelona, Valencia, Granada, Oviedo, Illes Balears y Murcia las más representadas, llegando a explicar aproximadamente el $75 \%$ de las firmas.

En este caso, sí hay diferencias profundas con los Congresos de la SEPJF (López, Seijo y Amado, 2017), al menos en lo que hace referencia a países extranjeros más representados (Portugal y México explican un 50\%, tras ellos con valores inferiores al 10\% Alemania, Colombia, Francia, Argentina, EE.UU., Palestina y Reino Unido). Más similitudes se dan en el caso de las instituciones españolas, donde tienen un mayor número de contribuciones las Universidades de Santiago de Compostela (37 autorías, 10,8\%), Valencia (36 autorías, 10,3\%) Barcelona (34 autorías, 9,9\%), Oviedo (33 autorías, 9,6\%), Granada (31 autorías, 9,1\%), Murcia (30 autorías, 8,7\%) o el Col-legi Oficial de Psicòlegs de Catalunya (23 autorías, 6,7\%).

El carácter más femenino que masculino de la revista queda reforzado por el hecho de que más de la mitad de los artículos están firmados en primer lugar por una mujer (58 de 104, 55,76\%), dos de ellos como firmante única, apareciendo en algo menos de un tercio (36 de 104, 34,61\%) como última firmante, pero solo en 16 casos en artículos 
firmados en primer lugar por un hombre, mientras que en 34 artículos firmados en primer lugar por mujeres es un hombre el que aparece como último firmante. Si entendemos que el criterio de firma no es el orden alfabético, sino la relevancia de la participación, estamos en un ámbito donde las mujeres, investigadoras y/o profesionales, ocupan un destacado lugar.

Son datos coincides con los procedentes de los congresos de la Sociedad (López et al., 2017; Velasco, Amado y Arias, 2014). Las mujeres firmaron significativamente más artículos que los hombres, y ocuparon de forma destacada las posiciones más relevantes de firma, como primer autor.

\subsection{Colaboración}

De los 104 documentos publicados en The EJPALC el 93,26\% (97 artículos) han sido firmados en colaboración por dos o más autores, mientras que solamente el 6,74\% (7 artículos) han sido firmados por un único autor. La media de firmas por trabajo durante todo el período es de 3,27. En la Tabla 3 se presentan la distribución por año del número firmas y la media de firmas por trabajo de lo publicado en la revista.

\begin{tabular}{lccccc}
\hline Años & $\begin{array}{c}\text { Trabajos de } \\
\text { 1 solo autor }\end{array}$ & $\begin{array}{c}\text { Trabajos } \\
\text { coautoría }\end{array}$ & $\begin{array}{c}\mathbf{N}^{\mathbf{0}} \\
\text { firmas }\end{array}$ & Media firmas/ artículo & $\begin{array}{c}\mathbf{N}^{\mathbf{0}} \text { máximo de } \\
\text { autores/trabajo }\end{array}$ \\
\hline $\mathbf{2 0 0 9}$ & 1 & 10 & 33 & 3,00 & 4 \\
\hline $\mathbf{2 0 1 0}$ & 0 & 10 & 32 & 3,20 & 6 \\
\hline $\mathbf{2 0 1 1}$ & 0 & 10 & 43 & 4,30 & 8 \\
\hline $\mathbf{2 0 1 2}$ & 1 & 9 & 30 & 3,00 & 5 \\
\hline $\mathbf{2 0 1 3}$ & 0 & 10 & 34 & 3,40 & 5 \\
\hline $\mathbf{2 0 1 4}$ & 1 & 10 & 32 & 2,91 & 5 \\
\hline $\mathbf{2 0 1 5}$ & 2 & 8 & 30 & 3,00 & 6 \\
\hline $\mathbf{2 0 1 6}$ & 0 & 11 & 31 & 2,82 & 4 \\
\hline $\mathbf{2 0 1 7}$ & 1 & 10 & 38 & 3,45 & 5 \\
\hline $\mathbf{2 0 1 8}$ & 1 & 9 & 38 & 3,80 & 6 \\
\hline Total & 7 & 96 & 341 & 3,27 & 8 \\
\hline
\end{tabular}

Tabla 3. Distribución por año del número de firmas por trabajo

Hay que mencionar que los trabajos publicados siguen la tendencia de otras áreas científicas, en las que se observa que el número de autores de los trabajos publicados en revistas científicas y técnicas aumenta de forma continuada y que predominan los trabajos realizados en colaboración. Sin embargo, la media de firmas/trabajo es inferior a la existente en revistas de otras disciplinas científicas (Yegros-Yegros, Tur y Amat, 2012), aunque no de forma generalizada, ya que se observa que, por ejemplo, supera el obtenido en el caso de la Psicología Educativa en España durante los quinquenios 20042008 y 2009-2013 (Osca-Lluch y González-Sala, 2017) y el de la revista Psicothema (González-Alcaide et al., 2010).

A la hora de analizar la colaboración científica de los autores (coautorías) a partir de la identificación de las principales relaciones de colaboración, se han construido las diferentes redes o representaciones visuales de las colaboraciones entre autores e instituciones utilizando los programas Pajek (de Nooy, Mrvar y Batagelj, 2012; Mrvar y 
Batagelj, 2016) y Ucinet (Borgatti, Everett y Freeman, 2002; Borgatti, Everett y Johnson, 2013).

En la Figura 1 está representada la red de colaboración entre los autores. Los nodos representan los autores y las líneas que unen los diferentes nodos representan las colaboraciones entre ellos. El grosor de los nodos indica el mayor o menor número de trabajos que ha realizado un autor.

El análisis de coautorías ha permitido identificar 49 grupos de investigación. El mayor de los grupos está conformado por 22 investigadores vinculados entre sí de forma directa o a través de intermediarios. Este grupo está constituido por una serie de autores con gran producción, como Enrique Gracia y Marisol Lila de la Universidad de Valencia, y Francisco Javier Rodríguez-Díaz y Juan Herrero de la Universidad de Oviedo. Enrique Gracia y Marisol Lila destacan también junto a Juan Herrero (Universidad de Oviedo), por su papel de intermediación dentro del grupo. Dentro de este grupo se distinguen tres subgrupos conectados a través de estos tres autores. La mayor intensidad de colaboración se produce entre Enrique Gracia y Marisol Lila, con 6 trabajos conjuntos. Lila y Gracia firman habitualmente conjuntamente y con colaboradores de la Universidad de Valencia, y en algún caso también de la Universidad de Oviedo a través de Juan Herrero.

El segundo grupo que destaca por tener un mayor número de componentes está integrado por 16 investigadores, entre los que se encuentran varios autores de diferentes universidades españolas que destacan por su productividad en esta disciplina, como son Ramón Arce (Universidad de Santiago de Compostela), Francisca Fariña (Universidad de Vigo), Fernando Jiménez (Universidad de Salamanca), Alfonso Palmer (Universitat Illes Balears), Guadalupe Sánchez (Universidad de Salamanca) y Manuel Vilariño (Universidad de Santiago de Compostela). Dentro de este grupo destacan por su papel de intermediación Barbara G. Amado (Universidad de Santiago de Compostela), Alfonso Palmer (Universitat Illes Balears) y Manuel Vilariño (Universidad de Santiago de Compostela). En este grupo, la mayor colaboración se ha producido entre Ramón Arce, Francisca Fariña y Manuel Vilariño, entre Fernando Jiménez (Universidad de Salamanca), Amada Ampudia (Universidad Nacional Autónoma de México) y Guadalupe Sánchez (Universidad de Salamanca), y entre Alfonso Palmer y Elena Gervilla, ambos de la Universitat Illes Balears. 


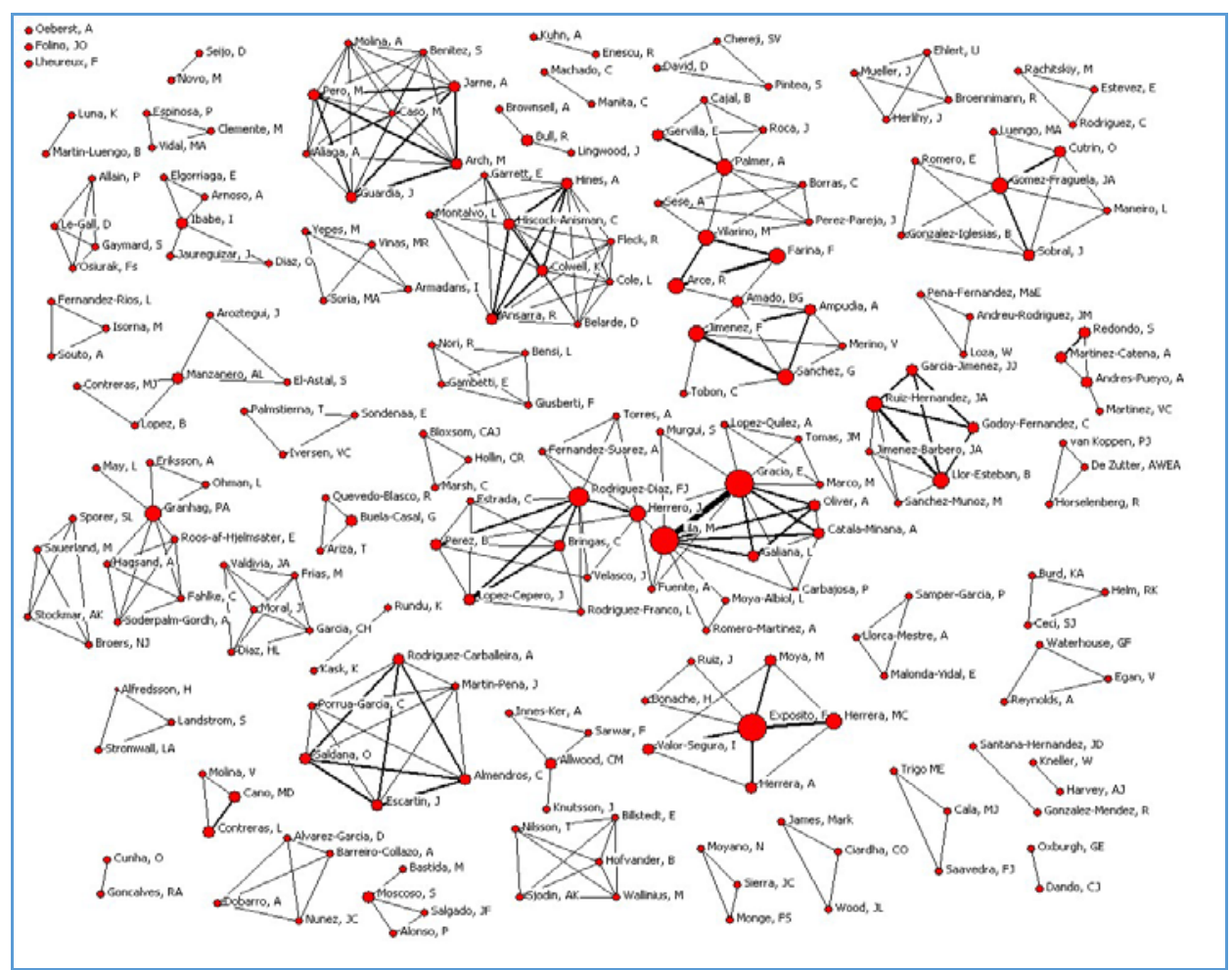

Figura 1. Redes de autoría en The European Journal of Psychology Applied to Legal Contex (2009-2017)

Se ha identificado un grupo compuesto por 9 autores, en el que no se observa ningún autor destacado, en cuanto que todos ellos están conectados entre sí con una misma intensidad de colaboración. Se han identificado también dos grupos compuestos cada uno por 8 investigadores, uno de ellos liderado por Pär Anders Granhag (Universidad de Gothenburg), que destaca también por su papel de conexión dentro de su grupo y dos grupos compuestos por 7 y 6 investigadores cada uno de ellos. En uno de los grupos formados por 7 investigadores, destacan las figuras de Francisca Expósito y Carmen Herrera y en otro, la figura de José Antonio Gómez-Fragüela. Hay que destacar la existencia de cuatro grupos formados por cinco investigadores cada uno de ellos, 9 grupos formados por 2 y 4 investigadores, siendo los grupos más frecuentes (18 grupos), los formados por 3 investigadores. Tal como se observa en la Figura 1, hay 3 nodos aislados, que representan a los tres autores que no han publicado ningún trabajo en coautoría en esta revista.

A efectos de facilitar la interpretación de los datos y hacer más legibles las relaciones entre los diferentes autores, se ha realizado la representación gráfica de una red con los autores que han publicado solamente 2 ó más trabajos en la revista durante el período 2009-2017 (Figura 2). En este caso, se ve claramente que el número de grupos es mucho menor, habiendo solamente 10 grupos de investigación. 


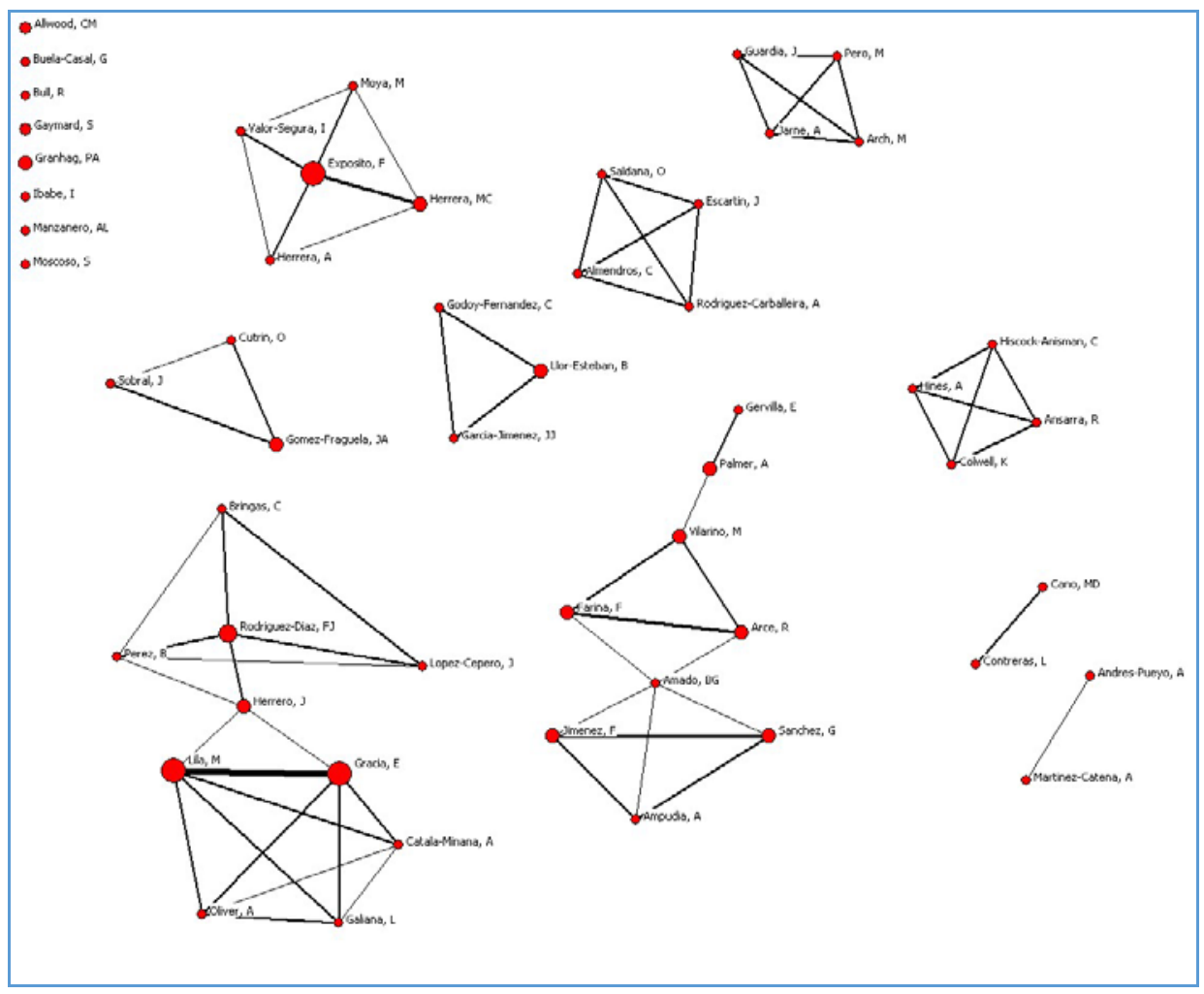

Figura 2. Redes de autoría de investigadores que han publicado 2 ó más trabajos

Se observa la existencia de 8 nodos aislados, que representan a los autores que han publicado dos o más trabajos realizados con autores con los que han firmado solamente un trabajo y que, por esta razón, no han sido representados en este gráfico. Se observa claramente que el grupo que está formado por un mayor número de investigadores es, de nuevo, el liderado por Enrique Gracia y Marisol Lila, y Francisco Javier RodríguezDíaz y Juan Herrero, pero que, en este caso, vemos que el grupo está formado por 11 investigadores. El segundo grupo en número sigue siendo también el constelado en torno a Ramón Arce, Francisca Fariña, Fernando Jiménez, Alfonso Palmer, Guadalupe Sánchez y Manuel Vilariño, pero en este caso, el grupo está formado por 9 investigadores.

\subsection{Temas principales}

En relación con el contenido de los artículos publicados en The EJPALC, se parte del sistema de categorías, y criterios, propuestos para el análisis de los trabajos recogidos en las Actas de los Congresos de la SEPJF (López et al., 2017; Velasco et al., 2014).

En la Tabla 4 se recoge la distribución de artículos publicados en la revista por categorías, junto a la de los trabajos recogidos en las Actas de los primeros XI congresos de la Sociedad Española de Psicología Jurídica y Forense, para facilitar análisis comparativos, aunque se modifica por el carácter diferencial del objeto de análisis. Puede apreciarse que el núcleo dominante es el de Evaluación Psicológica y Psicología del Testimonio (39,05\% en EJPALC y $15,31 \%$ en los congresos). En la revista explica casi dos quintas partes de los artículos, mientras que en los Congresos ocupa el tercer lugar tras Psicología Jurídica de la familia y violencia de género, esta última la segunda categoría con más documentos en la revista; en cambio existe una 
diferencia en el caso de la Psicología Jurídica de la familia, el área con más trabajos en los congresos, y la que menos tiene en la revista dos primeras también con fuerte presencia en la revista. No obstante, en general hay una razonable correspondencia entre ambas fuentes de datos.

El tema de la evaluación concita numerosos autores, de los que el más prolífico es Álvaro Rodríguez-Carballeira de la Universidad de Barcelona, junto a otros firmantes de la Universidad Autónoma de Madrid y de la Universidad de Zaragoza. Testimonio es el área que más autores no españoles atrae, y dentro de España destacan Ramón Arce y Francisca Fariña y autores de su grupo, sobre todo en la Universidad de Santiago de Compostela, Francisca Expósito y otros autores centrados en la Universidad de Granada, y Antonio Manzanero, director del grupo oficial de investigación sobre el tema de la Universidad Complutense. En menor proporción destacan los grupos en torno a Izaskun Ibabe (Universidad del País Vasco) que trabaja violencia hacia los padres, otro alrededor de Elena Gervilla y Alfonso Palmer (Universitat Illes Balears) que trabajan sobre decisión de ingerir cannabis y cocaína en adolescentes y el valor predictivo de ciertas variables para explicar el consumo de alcohol en población adolescente, y, también, el grupo de Lourdes Contreras (Universidad de Jaén) sobre violencia filio-parental y factores que inciden sobre la reincidencia delictiva de menores.

\begin{tabular}{lcccc}
\hline & $\begin{array}{c}\text { The European Journal of } \\
\text { Psychology Applied to Legal } \\
\text { Context }\end{array}$ & \multicolumn{2}{c}{$\begin{array}{c}\text { Libros de Actas de los } \\
\text { Congresos de la SEPJF }\end{array}$} \\
\hline Áreas & Número trabajos & $\%$ & Número trabajos & $\%$ \\
\hline Psicología Jurídica del Menor & 11 & 10,60 & 121 & 14,04 \\
\hline Violencia de Género & 18 & 17,30 & 139 & 16,12 \\
\hline Psicología Jurídica de la Familia & 3 & 2,90 & 146 & 16,94 \\
\hline Psicología Penitenciaria y Policial & 8 & 7,70 & 107 & 12,41 \\
\hline Victimología & 7 & 6,73 & 46 & 5,34 \\
\hline Psicología Jurídica y Forense & 5 & 4,80 & 111 & 12,88 \\
\hline Evaluación Psicológica y Testimonio & 41 & 39,42 & 132 & 15,31 \\
\hline No clasificados & 11 & 10,60 & 60 & 6,96 \\
\hline TOTAL & 104 & 100 & 862 & 100 \\
\hline
\end{tabular}

Tabla 4. Ámbitos temáticos de los artículos publicados en The European Journal of Psychology Applied to Legal Context y en los XI congresos de la Sociedad Española de Psicología Jurídica y Forense

Se complementa el análisis mediante una aproximación cuantitativa a los descriptores elegidos por los autores de los artículos. Existe una diversidad muy elevada, hasta 432 descriptores (4 diferentes por artículo), lo cual indica que no existe un acuerdo generalizado entre los autores a la hora de seleccionar descriptores para describir su trabajo concreto, aunque forma parte de un perfil de trabajos bien definido. 364 descriptores aparecen una sola vez, el 84,26\%, 39 dos veces (9,03\%), y un pequeño número 3 ó más veces, 29 (6,71\%). Existe, en cualquier caso, una coincidencia entre los descriptores más utilizados y los temas más tratados. Descriptores como simulación, testimonio, evaluación forense, MMPI-2, memoria de testigos, credibilidad o engaño estarían relacionados con artículos publicados en el tema de evaluación psicológica y testimonio, dominante en la revista. Las otras grandes áreas, "psicología jurídica del 
menor", "violencia de género", y "psicología jurídica de la familia" contendrían documentos con descriptores como violencia de pareja, adolescencia, actitudes, sexismo, crimen, delincuencia, empatía, psicopatología, comportamiento antisocial, menores delincuentes, reincidencia, agresión o violencia contra la mujer. El descriptor de "meta análisis" se incluye en diferentes artículos, como el de Amado, Arce y Fariña (2015), que realiza una revisión meta-analítica sobre el análisis de contenido del testimonio basado en criterios (hipótesis Undeutsch); en el de Alonso, Moscoso y Salgado (2017) sobre la entrevista conductual estructurada como garantía legal para garantizar la igualdad de oportunidades de empleo para las mujeres; en el de Chereji, Pintea y David (2012) al revisar la relación entre las distorsiones cognitivas y la ira en la conducta violenta, o en el de Alfaro, Puente, da Costa, Ruvalcaba y Páez (2018) que analizan estudios de América del Norte y del Sur, Europa y Oceanía sobre la asociación entre el temor al crimen y el bienestar subjetivo.

\subsection{Artículos con mayor visibilidad}

En la colección principal de la WoS se aprecia un promedio de citas por artículo de 8,90 , y en todas las bases de datos que se pueden consultar a través de la plataforma WoS el promedio es de 9,50. Si nos centramos en los trabajos citados, se observa que un $97,14 \%$ de los artículos han sido citados al menos una sola vez, de estos $6(5,71 \%)$, solo 3 no han recibido cita alguna ni en la colección principal, ni en todas las bases de datos. Estamos, pues, ante una revista que en general publica artículos que interesan a la comunidad científica, si bien aparece la habitual distribución diferencial.

En la media o por debajo encontramos, en todas las bases de datos 67 artículos, un $63,81 \%$. Por encima 38, 36,19\%, pero que explican un $71,19 \%$ de las citas. En la media o por debajo encontramos, en la colección principal 67 artículos, un 63,81\%. Por encima $38,36,19 \%$, pero que explican un $63,27 \%$ de las citas.

La Tabla 5 ofrece los diez artículos más citados en la colección principal, explicando casi un tercio del total de citas $(29,62 \%), 30$ citas por artículo de promedio. Con 13 artículos más llegamos a explicar el 52,71\% de las citas, más de la mitad de las citas con algo más de la quinta parte de los artículos (21,90\%). Estos 23 artículos tienen una media de 23,22 citas por artículo. Los 82 documentos restantes $(78,10 \%)$ explican solo un $47,29 \%$ de las citas, con un promedio de 12,35 citas.

En todas las bases de datos los resultados son análogos. Los diez artículos más citados reciben un $30,56 \%$ de las citas, y los 24 primeros llegan hasta el 54,48\%, con unas medias de citas por artículo de 33,10 y 21,25. Los restantes documentos tendrían una media de firmas de 6,21.

\section{CONCLUSIONES}

El desarrollo y consolidación de la disciplina de Psicología Jurídica en España ha sido un proceso lento que experimentó un gran desarrollo en la década de los 80 (Fariña, Arce y Novo, 2005). La Psicología Jurídica ha sido entendida tradicionalmente como la aplicación de la ciencia psicológica al Derecho, pero es mucho más. La Psicología Jurídica no solo cuenta con una parte aplicada, sino que también es capaz de elaborar teorías, metodologías que condicionan las actuaciones propias del Derecho y 
comprende más allá de éste, al abordar aspectos relevantes y relacionados con el comportamiento humano previo a la comisión de un acto delictivo (Arce, 2005).

\begin{tabular}{llc}
\hline \multicolumn{1}{c}{ Artículos } & \multicolumn{1}{c}{$\begin{array}{c}\text { Citas en colección } \\
\text { principal de WOS* }\end{array}$} & $\begin{array}{c}\text { Citas en todas las } \\
\text { bases de datos }\end{array}$ \\
\hline $\begin{array}{l}\text { 1. Predicting success indicators of an intervention } \\
\text { programme for convicted intimate-partner violence } \\
\text { offenders: The Contexto Programme }\end{array}$ & 31 & 50 \\
\hline $\begin{array}{l}\text { 2. Undeutsch hypothesis and criteria based content } \\
\text { analysis: A meta-analytic review }\end{array}$ & 33 & 36 \\
\hline $\begin{array}{l}\text { 3. In search of psychosocial variables linked to the } \\
\text { recidivism in young offenders }\end{array}$ & 31 & 39 \\
\hline $\begin{array}{l}\text { 4. Perceived neighborhood social disorder and attitudes } \\
\text { toward domestic violence against women among Latin- } \\
\text { American inmigrants. }\end{array}$ & 30 & 35 \\
\hline $\begin{array}{l}\text { 5. Psychological adjustment and victim-blaming among } \\
\text { intimate partner violence offenders: the role of social } \\
\text { support and stressful life events. }\end{array}$ & 29 & 31 \\
\hline $\begin{array}{l}\text { 6. Adolescent violence against parents: Is it a } \\
\text { consequence of gender inequality? }\end{array}$ & 28 & 28 \\
\hline $\begin{array}{l}\text { 7. Behavioral problems and depressive symptomatology } \\
\text { as predictors of child-to-parent violence }\end{array}$ & 25 & 26 \\
\hline $\begin{array}{l}\text { 8. Pathological publishing: A new psychological disorder } \\
\text { with legal consequences? }\end{array}$ & 23 & 23 \\
\hline $\begin{array}{l}\text { 9. Intimate partner violence offenders: Generating a data- } \\
\text { based typology of batterers and implications for treatment }\end{array}$ & 23 & 26 \\
\hline $\begin{array}{l}\text { 10. Child-to-parent violence: The role of exposure to } \\
\text { violence and its relationship to social-cognitive processing }\end{array}$ & & 37 \\
\hline
\end{tabular}

Tabla 5. Artículos más citados en The European Journal of Psychology Applied to Legal Context

Los datos confirman la afirmación (Arch, Pereda, Jarne-Esparcia, Andrés y GuàrdiaOlmos, 2010; González-Sala, Osca-LLuch, Tortosa-Gil y Peñaranda-Ortega, 2017; Quevedo-Blasco et al., 2012). Se ha incrementado el número de revistas listadas en WoS y Scopus, así como el de artículos e investigadores sobre el tema. Es más, ha crecido significativamente el índice de colaboración y el número de grupos emergentes especializados, como el volumen de publicaciones en revistas de carácter general y multidisciplinar.

Consistente con la literatura publicada se aprecia que existe un grupo reducido de investigadores muy activos, responsable de buena parte de la producción científica en el ámbito jurídico-forense, aspecto ya apuntado por López et al. (2017) y Quevedo-Blasco et al. (2012). Grupo que, además, muestra un elevado nivel de colaboración, consonante con el general, que se sitúa en más de tres firmas de media, llegando hasta 8 el máximo de autoría conjunta, en línea con la tendencia existente en la Psicología española (Fundación Española para la Ciencia y la Tecnología, 2017). El análisis de coautoría descubre la existencia de varios grupos de investigación consolidados, próximos al equipo editorial y a la Sociedad Española de Psicología Jurídica y Forense de la que es órgano de expresión, al igual que ocurre con los autores más productivos. De igual forma, se descubre un eje vertebrador institucional que incluye las Universidades de Valencia, Santiago de Compostela, Oviedo, A Coruña, Vigo, Granada, Sevilla, Barcelona y Murcia, que vincula también universidades extranjeras, sobre todo mejicanas (López et al., 2017; Velasco et al., 2014).

Una singularidad importante de la revista, al igual que ocurre en los Congresos de la Sociedad (López et al., 2017), es la elevada presencia de mujeres en las publicaciones, su activa productividad, su posicionamiento en el orden de firma, y su impacto diferencial en la comunidad científica, y en el Consejo Editorial (González-Sala y OscaLluch, 2018). Define una revista muy sensible al género frente a la situación general del 
ámbito donde los hombres doblan a las mujeres en producción de documentos y firman más en primera posición (Barrios, Villarroya y Borrego, 2013; González-Alcaide et al., 2010; Torres-Salinas y Muñoz-Muñoz, 2011; Torres-Salinas, Muñoz-Muñoz y JiménezContreras, 2011; Sierra, Buela-Casal, Bermúdez y Santos, 2009; Velasco, Vilariño, Amado y Fariña, 2014).

Los intereses de los autores se centran en los temas de evaluación psicológica, credibilidad y testimonio y la violencia de género principalmente, intereses que se siguen manteniendo en números posteriores, como es el caso de la violencia de género en los trabajos de Juarros-Basterretxea, Herrero, Fernández-Suarez, Pérez, y RodríguezDíaz (2018), el de Martín-Fernández et al. (2018) o el de Loinaz, Marzabal y AndrésPueyo (2018). Un espectro muy representativo de las aplicaciones de la Psicología al contexto legal (López et al., 2017; Morales-Quintero y López-García, 2010; QuevedoBlasco et al., 2012). Finalmente, destacar, por ser el objetivo de la revista, la contribución a la transferencia de conocimiento con la creación de instrumentos de medida (e.g., Saldaña, Rodríguez-Carballeira, Almendros y Escartín, 2017); herramientas forenses (e.g., Vilariño, Arce y Fariña, 2013) y metodológicas (e.g., Salgado, 2018).

En relación a la visibilidad y repercusión de los trabajos, hay que destacar que el 95\% de los trabajos publicados han sido citados, con un promedio de 8 citas por artículo. Siete artículos explican una cuarta parte del total de citas, y con 12 más llegamos a explicar el $50,61 \%$ de las citas. Esta elevada citación, unido al poco significativo número de autocitas, muestra el impacto internacional de los artículos, ocupando un lugar muy destacado en la producción citada en el ámbito (Osca-Lluch, González-Sala y Tortosa, 2017).

The EJPALC juega un papel fundamental en el posicionamiento y la internacionalización de la Psicología Jurídica española (Osca, Tortosa, González, y Tortosa, 2017), pero también de la Psicología española, cada vez más tenida en cuenta en el ámbito (Tortosa, Osca, López, y Alfaro, 2019). Muy positivo en el proceso resultó la firma, el 15 de febrero de 2013, de un acuerdo de colaboración para la coedición de la revista con el COP Madrid. Es la revista oficial de la Sociedad Española de Psicología Jurídica y Forense y de la Asociación Iberoamericana de Justicia Terapéutica. Desde su inicio la revista destaca por el cumplimiento de los indicadores de calidad editorial y su grado de apertura, medido en relación con la composición de sus comités editoriales y consejos de redacción, así como a través de la procedencia profesional y nacional de los autores que publican en ella. Como destaca Arce, entre las revistas de referencia mundial, The EJPALC ha alcanzado el percentil 96 en su categoría quedando por encima de las revistas de Asociaciones Profesionales como la American Psyhcological Association o la Bristish Psychological Association, o la European Association of Psyhology and Law (Anónimo, 2018).

El cumplimiento de los indicadores de calidad, su decidida apuesta por el inglés, así como el interés provocado por sus contenidos, es lo que ha llevado a posicionar la revista en los primeros puestos de los rankings de citas internacionales de referencia en un tan breve período de tiempo. De hecho, se trata de la única revista especializada editada en España que no sólo satisface el criterio más estricto (Q1 en el JCR) de evaluación de la Comisión Nacional Evaluadora de la Actividad Investigadora (CNEAI), encargada de evaluar períodos sexenales de actividad investigadora de los profesores universitarios y del personal de las escalas científicas del CSIC, sino que 
estaría en una categoría superior, el Top 10\%, que recoge cerca del $90 \%$ del impacto total (Infocop, 2018).

Es actualmente la única revista editada en un país iberoamericano que ocupa los primeros rangos tanto en JCR como en SJR. Es la única opción nacional que tienen los especialistas de la materia para publicar trabajos que les sirvan para que se reconozcan sus méritos académicos y/o investigadores. Muchos derivan sus trabajos a revistas de carácter general o multidisciplinar, lo que contribuye a restar visibilidad a los trabajos o que estos pasen desapercibidos para el resto de especialistas en la materia. Todo ello convierte The EJPALC en referente para quienes buscan la información en revistas especializadas. Sin duda forma parte del reducido grupo de referentes que es sensible a los nuevos cambios que debe afrontar la disciplina en este a la vez globalizado y parcializado siglo XXI (Arce y Fariña, 2013).

\section{BIBLIOGRAFÍA}

Aguado-López, E. y Becerril-García, A. (2016). ¿Publicar o perecer? El caso de las ciencias sociales y las humanidades en Latinoamérica. Revista Española de Documentación Científica, 39 (4), e151. https://doi.org/10.3989/redc.2016.4.1356

Alfaro, E., Real, S., Tortosa, F. y Jólluskin, G. (2004). El desarrollo académico de la psicología jurídica. Anuario de Psicología Jurídica, 14, 99-114. http://journals.copmadrid.org/apj/archivos/93973.pdf

Alfaro, L., Puente, A., da Costa, S., Ruvalcaba, N. y Páez, D. (2018). Effects of fear of crime on subjective Well-being: A meta-analytic review. European Journal of Psychology Applied to Legal Context, 10 (2), 89-96. https://doi.org/10.5093/ejpalc2018a9

Alonso, P., Moscoso, S. y Salgado, J.F. (2017). Structured behavioral interview as a legal guarantee for ensuring equal employment opportunities for women: A meta-analysis. European Journal of Psychology Applied to Legal Context, 9 (1), 15-23. https://doi.org/10.1016/j.ejpal.2016.03.002

Amado, B.G., Arce, R. y Fariña, F. (2015). Undeutsch hypothesis and criteria based content analysis: A meta-analytic review. European Journal of Psychology Applied to Legal Context, 7 (1), 3-12. https://doi.org/10.1016/j.ejpal.2014.11.002

Anónimo (2013). El COP Madrid potencia las publicaciones científico-profesionales del área de psicología jurídica. Infocop online. Recuperado de: http://www.infocop.es/view_article.asp?id=4554

Anónimo (2018). Cinco revistas españolas de Psicología, entre las primeras con mayor factor de impacto según el JCR. Infocop online. Recuperado de:

http://www.infocop.es/view_article.asp?id=7636

Arce, R. (2005). La construcción de la psicología jurídica en Europa y su estatus actual. En R. Abrunhosa y C. Machado (Eds.). Manual de psicología jurídica (pp. 103-114). Coimbra, Portugal: Quarteto. Recuperado de: https://www.researchgate.net/publication/275536227_La_construccion_de_la_Psicologia_j uridica_en_Europa_y_su_estatus_actual

Arce, R. y Fariña, F. (2013). Psicología social aplicada al ámbito jurídico. En A. V. Arias, J. F. Morales, E. Nouvilas, y J. L. Martínez-Rubio (Coords.). Psicología social aplicada (pp. 157-181). Madrid, España: Editorial Médica Panamericana. Recuperado de: http://www.usc.es/export9/sites/webinstitucional/gl/servizos/uforense/descargas/Psicologia _aplicada_al_ambito_juridico.pdf

Arch, M., Pereda, N., Jarne-Esparcia, A., Andrés, A. y Guardia-Olmos, J. (2010). Producción científica de la psicología forense en España: Un estudio bibliométrico. Revista 
Iberoamericana de Psicología y Salud, 1 (2), 155-166. Recuperado de: http://www.redalyc.org/articulo.oa? id $=245116406003$

Barrios, M., Villarroya, A. y Borrego, A. (2013). Scientific production in psychology: A gender analysis. Scientometrics, 95 (1), 15-23. https://doi.org/10.1007/s11192-012-0816-4

Borgatti, S.P., Everett, M.G. y Freeman, L.C. (2002). Ucinet for Windows: Software for social network analysis. Harvard, Mass, USA: Analytic Technologies. Recuperado de: https://www.researchgate.net/publication/216636663

Borgatti, S.P., Everett, M.G. y Johnson, J.C. (2013). Analyzing social networks. London: SAGE. https://pdfs.semanticscholar.org/c0ab/40a9baec1c8ca54b96ec33a2957757f00bf5.pdf

Buela-Casal, G., Guillén-Riquelme, A., Ramiro-Sánchez, T. y Quevedo-Blasco, R. (2017). Ranking de investigación de las universidades públicas españolas. Revista Iberoamericana de Psicología y Salud, 8 (1), 21-35. https://doi.org/10.23923/j.rips.2017.08.003

Carpintero, H. (2006). Breve historia de la psicología jurídica. En E. Garrido, J. Masip, y M.C. Herrero (Eds.). Psicología jurídica (pp. 43-75). Madrid: Pearson Prentice-Hall.

Chereji, S.V., Pintea, S. y David, D. (2012). The relationship of anger and cognitive distortions with violence in violent offenders' population: A meta-analytic review. European Journal of Psychology Applied to Legal Context, 4 (1), 59-77.

https://journals.copmadrid.org/ejpalc/art/ed277964a8959e72a0d987e598dfbe72

Colegio Oficial de Psicólogos. (1998). Perfiles profesionales del psicólogo. Madrid: Autor. Recuperado de: https://www.cop.es/perfiles/

De Nooy, W., Mrvar, A. y Batagelj, V. (2012). Exploratory social network analysis with Pajek. Revised and expanded second edition. Cambridge, NJ: Cambridge University Press.

Fariña, F., Arce, R. y Jólluskin, G. (2000). Psicología y ley: Notas sobre una realidad reciente. Revista de Historia de la Psicología, 21 (2-3), 529-542. Recuperado de: https://www.revistahistoriapsicologia.es/revista/2000-vol-21-n\%C3\%BAm-2-3/

Fariña, F., Arce, R. y Seijo, M. (2005). Historia de la psicología jurídica en América y Europa. En R. Arce, F. Fariña, y M. Novo (Eds.). Psicología jurídica (pp. 29-44). Santiago de Compostela: Xunta de Galicia. Recuperado de: https://www.researchgate.net/profile/Ramon_Arce/publication/288670812_Psicologia_juri dica/links/5683079b08aebccc4e0e1f6e/Psicologia-juridica.pdf

Fariña, F., Arce, R. y Novo, M. (2005). Notas sobre la Historia de la psicología jurídica en España. En R. Arce, F. Fariña, y M. Novo (Eds.). Psicología jurídica (pp. 45-55). Santiago de Compostela: Xunta de Galicia. Recuperado de:

https://www.researchgate.net/profile/Ramon_Arce/publication/288670812_Psicologia_juri dica/links/5683079b08aebccc4e0e1f6e/Psicologia-juridica.pdf

Fundación Española para la Ciencia y la Tecnología. (2017). Indicadores bibliométricos de la actividad científica española 2005-2014. Edición 2016. Madrid: Fundación Española para la Ciencia y la Tecnología. Recuperado de:

https://www.fecyt.es/es/publicacion/indicadores-bibliometricos-de-la-actividad-cientificaespanola-2005-2014

González-Alcaide, G., Castelló-Cogollos, L., Bolaños-Pizarro, M., Alonso-Arroyo, A., Valderrama-Zurián, J.C. y Aleixandre-Benavent, R. (2010). Veinte años de investigación de la psicología española en Psicothema (1989-2008). Psicothema, 22 (1), 41-50. http://www.psicothema.com/pdf/3694.pdf

González-Sala, F., Osca-Lluch, J., Tortosa-Gil, F. y Peñaranda-Ortega, M. (2017). Characterization of legal psychology through psychology journals included in criminology \& penology and law categories of Web of Science. Anales de Psicología, 33 (2), 411-416. https://doi.org/10.6018/analesps.33.2.262591

González-Sala, F. y Osca-Lluch, J. (2018). Desigualdad de género en órganos directivos y producción científica de las revistas iberoamericanas de psicología de mayor visibilidad internacional. Revista Española de Documentación Científica, 41 (3), e211. https://doi.org/10.3989/redc.2018.3.1506 
González-Sala, F., Osca-Lluch, J., Tortosa-Gil, F. y Peñaranda-Ortega, M. (2018). Psicología Jurídica y Forense en España: Un estudio desde los másteres oficiales y títulos propios adscritos a las universidades españolas. Anuario de Psicología Jurídica, 28, 74-80. https://doi.org/10.5093/apj2018a3

Infocop (2018). Cinco revistas españolas de Psicología, entre las primeras con mayor Factor de impacto según el JCR. Infocop. http://www.infocop.es/view_article.asp?id=7636

Juarros-Basterretxea, J., Herrero, J., Fernández-Suarez, A., Pérez, B. y Rodríguez-Díaz, F.J. (2018). Are generalist batterers different from generally extra-family violent men? A study among imprisoned male violent offenders. European Journal of Psychology Applied to Legal Context, 10 (1), 8-14. https://doi.org/10.5093/ejpalc2018v10n1a1

Loinaz, I., Marzabal, I. y Andrés-Pueyo, A. (2018). Risk factors of female intimate partner and non-intimate partner homicides. European Journal of Psychology Applied to Legal Context, 10 (2), 49-55. https://doi.org/10.5093/ejpalc2018a4

López, A., Seijo, D. y Amado, B. (2017). Perfil bibliométrico de la psicología jurídica y forense en la "colección psicología y ley". En C. Bringas y M. Novo (Eds.). Psicología jurídica: Conocimiento y práctica (pp. 459-476). Colección Psicología y Ley, no. 14. Santiago de Compostela: Sociedad Española de Psicología Jurídica y Forense. Recuperado de: https://www.researchgate.net/publication/324605432_Coleccion_de_Psicologia_y_Ley_n_ 14_Psicologia_Juridica_Conocimiento_y_practica

Martín-Fernández, M., Gracia, E., Marco, M., Vargas, V., Santirso, F.A. y Lila, M. (2018). Measuring acceptability of intimate partner violence against women: Development and validation of the A-IPVAW Scale. European Journal of Psychology Applied to Legal Context, 10 (1), 26-34. https://doi.org/10.5093/ejpalc2018a3

Morales-Quintero, L.A. y López-García, E. (2010). Psicología Jurídica: Quehacer y desarrollo. Diversitas, 6 (2), 237-256. Recuperado de:

http://www.scielo.org.co/pdf/dpp/v6n2/v6n2a04.pdf

Mrvar, A. y Batagelj, V. (2016). Analysis and visualization of large networks with program package Pajek. Complex Adaptive Systems Modeling, 4 (6). https://doi.org/10.1186/s40294016-0017-8

Muñoz, J.M., Manzanero, A.L., Alcázar, M.A., González, J.L., Pérez, M.L. y Yela, M. (2011). Psicología jurídica en España: Delimitación conceptual, campos de investigación e intervención y propuesta formativa dentro de la Enseñanza Oficial. Anuario de Psicología Jurídica, 21 (1), 3-14. https://doi.org/10.5093/jr2011v21a1

Nicolás, L. de (1995). Perfil, rol y formación del psicólogo jurídico. Papeles del Psicólogo, 63, 53-68. http://www.papelesdelpsicologo.es/resumen?pii=692

Osca-Lluch, J. y González-Sala, F. (2017). Evolución de las redes científicas y grupos de investigación. El caso de la psicología educativa en España durante los quinquenios 20042008 y 2009-2013. Anales de Psicología, 33 (2), 356-364.

http://dx.doi.org/10.6018/analesps.33.2.249891

Osca-Lluch, J., González-Sala, F. y Tortosa, M. (2017). Visibilidad internacional e impacto de la producción científica española en psicología jurídica. En C. Bringas y M. Novo (Eds.). Psicología jurídica: Conocimiento y práctica (11-24). Colección Psicología y Ley, No 14. Santiago de Compostela: Sociedad Española de Psicología Jurídica y Forense. Recuperado de:

https://www.researchgate.net/publication/328791400_X_Congreso_InterNacional_de_Psic ologia_Juridica_y_Forense_Libro_de_actas

Osca-Lluch, J., Tortosa, M., González-Sala, F. y Tortosa, F. (2017). Indicadores de calidad de las publicaciones científicas en psicología jurídica. Acción Psicológica, 14 (2), 85-98. https://doi.org/10.5944/ap.14.2.20759

Quevedo-Blasco, R., Ariza, T. y Raya, L. (2012). Análisis de la producción de la psicología jurídica en España (1989-2010). Aula Abierta, 40 (2), 117-128. https://dialnet.unirioja.es/descarga/articulo/3921062.pdf 
Saldaña, O., Rodríguez-Carballeira, Á., Almendros, C. y Escartín, J. (2017). Development and validation of the Psychological Abuse Experienced in Groups Scale. European Journal of Psychology Applied to Legal Context, 9 (2), 57-64.

https://doi.org/10.1016/j.ejpal.2017.01.002

Santolaya, F., Berdullas, M. y Fernandez, J.R. (2001). The decade 1989-1998 in Spanish psychology: An analysis of development of professional psychology in Spain. The Spanish Journal of Psychology, 4 (2), 237-252. https://doi.org/10.1017/S1138741600005771

Salgado, J.F. (2018). Transforming the Area under the Normal Curve (AUC) into Cohen's d, Pearson's $\mathrm{r}_{\mathrm{pb}}$, Odds-Ratio, and Natural Log Odds-Ratio: Two conversion tables. European Journal of Psychology Applied to Legal Context, 10 (1), 35-47. https://doi.org/10.5093/ejpalc2018a5

Sierra, J.C., Buela-Casal, G., Bermúdez, M.P. y Santos, P. (2009). Diferencias por sexo en los criterios y estándares de productividad científica y docente en profesores funcionarios en España. Psicothema, 2 (1), 124-132. Recuperado de:

http://www.psicothema.com/pdf/3605.pdf

Torres-Salinas, D. y Muñoz-Muñoz, A. (2011). Análisis bibliométrico de la situación de las mujeres investigadoras de ciencias sociales y jurídicas en España. Revista Española de Documentación Científica, 34 (1), 11-28. http://dx.doi.org/10.3989/redc.2011.1.794

Torres-Salinas, D., Muñoz-Muñoz, A.M. y Jiménez-Contreras, E. (2011). Análisis bibliométrico de la situación de las mujeres investigadoras de ciencias sociales y Jurídicas en España. Revista Española de Documentación Científica, 34 (1), 11-28.

https://doi.org/10.3989/redc.2011.1.794

Tortosa, F., Civera, C., Fariña, F. y Alfaro, E. (2008). La primera singladura de la psicología jurídica en España. En F.J. Rodríguez, C. Bringas, F. Fariña, R. Arce y A. Bernardo (Eds.). Psicología jurídica: Familia y victimología (pp. 11-38). Oviedo: Ediciones de la Universidad de Oviedo. Recuperado de: http://gip.uniovi.es/T6EJD.pdf

Tortosa, M., Osca, J., López, W. y Alfaro, E. (2019). International positioning of the spanish psychology journals. Anales de Psicología, 35 (2), 332-340. http://dx.doi.org/10.6018/analesps.35.2.332171

Velasco, V., Amado, B.G. y Arias, E. (2014). Análisis bibliométrico de las publicaciones del congreso (Inter)Nacional de Psicología Jurídica y Forense. En R. Arce, F. Fariña, M. Novo, y D. Seijo (Eds.). Psicología jurídica y forense: Investigación y acción (pp. 3-13). Santiago de Compostela: Sociedad Española de Psicología Jurídica y Forense. http://dx.doi.org/10.13140/2.1.2655.3286

Velasco, B., Vilariño, M., Amado, B. y Fariña, F. (2014). Análisis bibliométrico de la investigación española en psicología desde una perspectiva de género. Revista Iberoamericana de Psicología y Salud, 5 (2), 105-118. https://doi.org/10.13140/2.1.3158.5924

Vilariño, M., Arce, R. y Fariña, F. (2013). Forensic-clinical interview: Reliability and validity for the evaluation of psychological injury. European Journal of Psychology Applied to Legal Context, 5 (1), 1-21.

https://journals.copmadrid.org/ejpalc/archivos/articulo20180219092235.pdf

Vilariño, M., Fariña, F. y Arce, R. (2009). Discriminating real victims from feigners of psychological injury in gender violence: Validating a protocol for forensic settings. European Journal of Psychology Applied to Legal Context, 1 (2), 221-243. Recuperado de: https://journals.copmadrid.org/ejpalc/art/98b418276d571e623651fc1d471c7811

Yegros-Yegros, A., Tur, E.M. y Amat, C.B. (2012). Número de autores y colaboración institucional en los originales de investigación biomédica española. Evolución de los valores básicos de referencia en el período 1990-2009. Medicina Clínica, 138 (4), 165-170. https://doi.org/10.1016/j.medcli.2011.02.006 